This item was submitted to Loughborough's Research Repository by the author.

Items in Figshare are protected by copyright, with all rights reserved, unless otherwise indicated.

\title{
Comparative study of nanomaterials' effective properties using canonical formations
}

PLEASE CITE THE PUBLISHED VERSION

http://dx.doi.org/10.1109/LAPC.2010.5666294

PUBLISHER

Loughborough University (@ IEEE)

VERSION

AM (Accepted Manuscript)

LICENCE

CC BY-NC-ND 4.0

REPOSITORY RECORD

Njoku, Chinwe C., W.G. Whittow, and J.C. Vardaxoglou. 2019. "Comparative Study of Nanomaterials' Effective Properties Using Canonical Formations”. figshare. https://hdl.handle.net/2134/9995. 
This item was submitted to Loughborough's Institutional Repository (https://dspace.lboro.ac.uk/) by the author and is made available under the following Creative Commons Licence conditions.

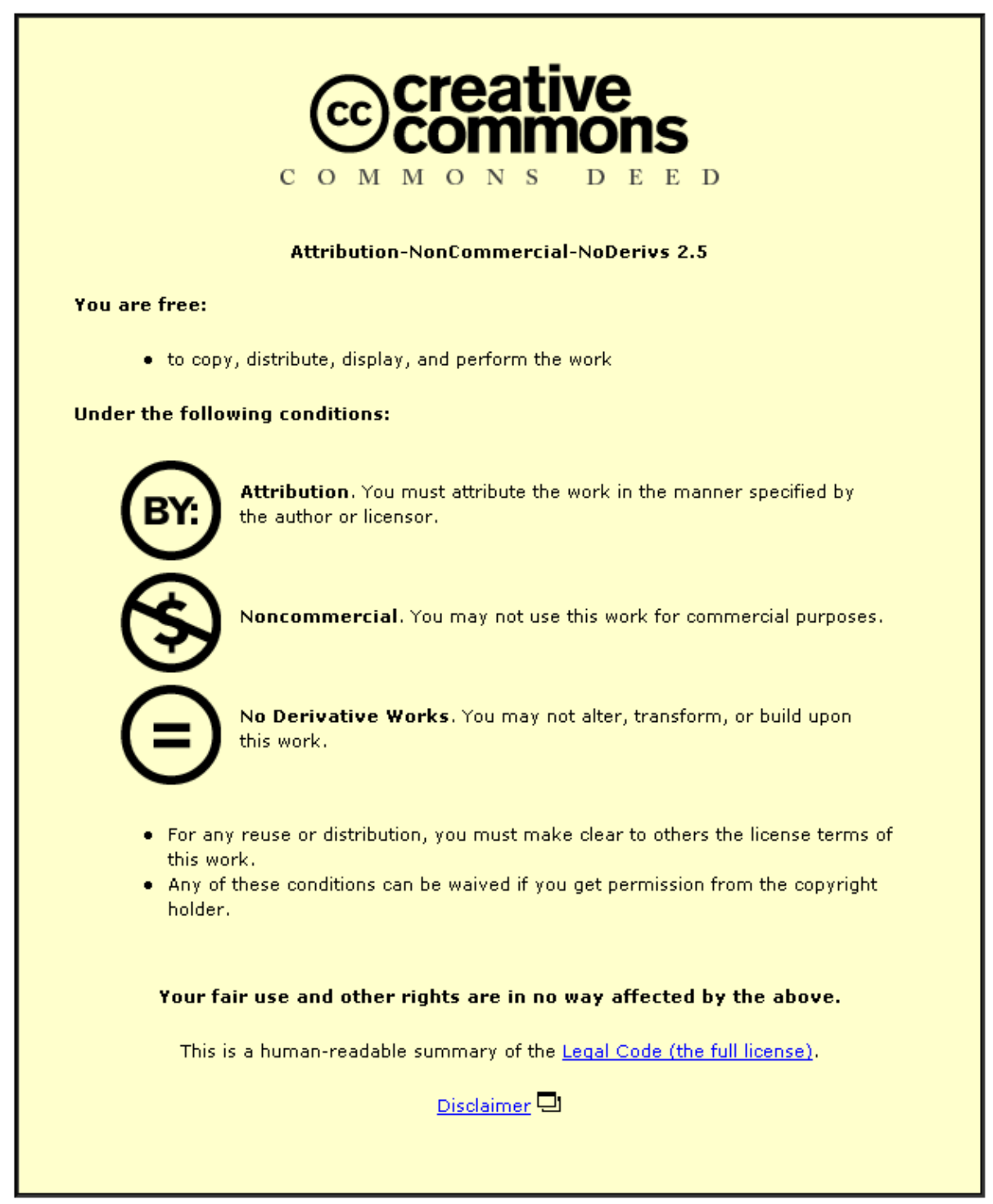

For the full text of this licence, please go to: http://creativecommons.org/licenses/by-nc-nd/2.5/ 


\title{
Comparative Study of Nanomaterials' Effective Properties Using Canonical Formations
}

\author{
C. C. Njoku ${ }^{\# 1}$, W. G. Whittow ${ }^{\# 2}$, J. C. Vardaxoglou ${ }^{\# 3}$ \\ ${ }^{\#}$ Electronic and Electrical Engineering Department, Loughborough University \\ Loughborough, LE11 3TU, UK \\ ${ }^{1} \mathrm{c} . c . n j o k u @ 1$ boro.ac.uk \\ w.g.whittow@lboro.ac.uk \\ j.c.vardaxoglou@lboro.ac.uk
}

\begin{abstract}
An analytical study of artificial materials created by embedding spherical nano-particles in an infinite homogenous medium is presented in this paper. Propositions by various contributors to this area of the nanomaterials' field have been compared with respect to their approaches in finding the effective constituent parameters, over wide frequency ranges. Their closed form equations were examined to check if their results were reproducible. Some of the main variables affecting the characteristics of these structures were varied individually to understand their role in determining the effective constituent parameters of the mixture. Finally, a choice was made on the most consistent proposition for examining structures created from nanomaterials.
\end{abstract}

\section{INTRODUCTION}

Since the work carried out by Lewin in 1946 on finding the electrical constants of a material loaded with spherical particles [1], considerable interest has been shown [2]-[10] in creating artificial materials that otherwise will not exist. Lord Rayleigh [2] appeared to be the first to examine how the properties of a medium are affected when obstacles are placed in it. However, more commonly cited and used for related problems is the analysis carried out by Lewin [1]. His analysis is a strong basis for the work carried out on using nanoparticles to build antennas and dielectrics without having to work with already established materials but 'creating' new materials that can provide the constitutive parameters, losses and other characteristics pre-determined for the whole antenna system to have. This method has the potential ultimately of integrating the whole antenna structure into one process.

Starting from Lewin's work one can formulate a basis for a combination of a variety of structures with bespoke electromagnetic (EM) parameters (notably highly conducting or highly dielectric) that can be integrated to form complete antennas and circuits. Fig. 1 above shows what our intended antenna structure might look like. Larger structures $(\sim \mathrm{mm})$ can be made by suitably arranging these extremely small nano-particles (metallic and/or non-metallic), thus allowing resonance at microwave frequencies to be possible. Fabrication and physical advantages include potentially faster fabrication processes and reduced production costs [10]. Using nanomaterial fabrication methods will enable novel and bespoke substrate properties, by controlling the size and volume ratio of the particles. Electromagnetic advantages

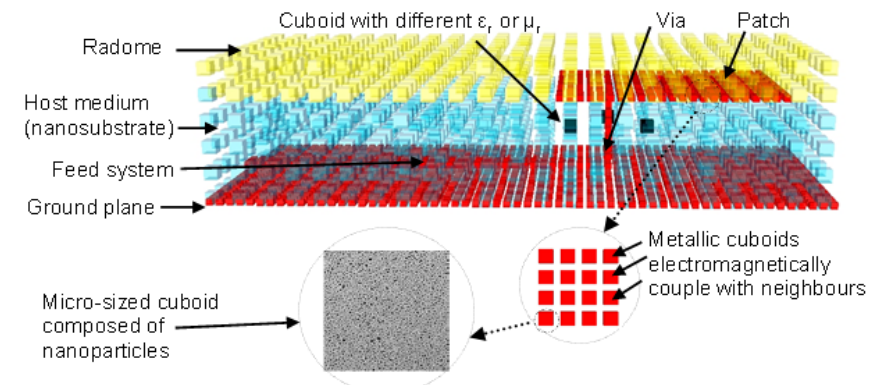

Fig. 1 A possible antenna structure composed of various nano-particles of different sizes and/or properties

(bandwidth, size and efficiency) can be achieved by varying the permittivity with the local electric field strength, or by creating substrates with equal permittivity and permeability.

This paper compares the analyses by various authors in this field, to get a canonical solution for one or more layers of differing characteristics, synthesizing their properties should the need for having a graded layer arise. This solution will form a starting point for further work to be done in achieving artificial materials suitable for microwave frequencies.

\section{THEORETICAL ANALYSIS REVIEW}

A general mathematical expression for electric permittivity, magnetic permeability, electrical and thermal conductivities, and particle diffusivity is given for particle-embedded mixtures in [3] as

$$
K_{m}=K_{2} \frac{\left[\left(K_{1}+2 K_{2}\right)\left(K_{2}-K_{1}\right)^{-1}\right]-2 p+C\left(K_{1}, K_{2}, p\right)}{\left[\left(K_{1}+2 K_{2}\right)\left(K_{2}-K_{1}\right)^{-1}\right]+p+C\left(K_{1}, K_{2}, p\right)}
$$

where $K_{1}, K_{2}$ and $K_{m}$ are the appropriate parameters of the inclusions, the host medium and the mixture respectively; $p$ is the total volume fraction of the spheres, and is given as described in the text below equation (2). $C\left(K_{1}, K_{2}, p\right)$ represents corrections for higher-order multipole terms as a result of the decomposition of the scattered field [1], [11]

\section{A. Lewin's Analysis [1]}

Lewin studied a homogenous medium within which were embedded spherical particles, with uniform spacing, (centreto-centre), $s$ and radius, $a$, in order to determine the effective permittivity $(\varepsilon)$ and permeability $(\mu)$ of the mixture. He did this by summing the total scattered field within the mixture 
using an approximation of Stratton's equation in [11], which deals with the scattering of electromagnetic waves by a sphere. The analysis assumes that the particles are uniformly spaced in a cubic lattice. The effective properties of the mixture are defined in (2) while those of the particle are defined in (3).

$$
\mu=\mu_{1}\left(1+\frac{3 f_{v}}{\frac{\mu_{p}+2 \mu_{1}}{\mu_{p}-\mu_{1}}-f_{v}}\right) ; \quad \varepsilon=\varepsilon_{1}\left(1+\frac{3 f_{v}}{\frac{\varepsilon p+2 \varepsilon_{1}}{\varepsilon_{p}-\varepsilon_{1}}-f_{v}}\right)
$$

where $(\varepsilon, \mu),\left(\varepsilon_{1}, \mu_{1}\right)$ and $\left(\varepsilon_{p}, \mu_{p}\right)$ are the permittivity and permeability of the mixture, host and particles respectively, and $f_{v}=\frac{4}{3} \pi a^{3} / s^{3}$ is the particle volume ratio. Also,

$$
\frac{\varepsilon_{p}}{\varepsilon_{2}}=\frac{\mu_{p}}{\mu_{2}}=\frac{2(\sin \theta-\theta \cos \theta)}{\left(\theta^{2}-1\right) \sin \theta+\theta \cos \theta}=F(\theta)
$$

where $\varepsilon_{2}$ and $\mu_{2}$ are the parameters of the particle's bulk material, $\theta=k_{0} a \sqrt{\mu_{2} \varepsilon_{2}}$, vacuum wave number, $k_{0}=2 \pi / \lambda$, and $\lambda$ is the free-space wavelength. The validity of his study is restricted to high frequencies as long as the size of the particles is much smaller than the wavelength and the particles are not densely packed.

\section{B. W. T. Doyle [4] and X. Cai et al's [5] Studies}

By decomposing the scattered field from an incident wave on a sphere into multipole terms [1], [4], and applying the correct boundary conditions at the surface of the sphere [11], the electric and magnetic scattering coefficients can be obtained. The $m$ th order electric and magnetic scattering coefficients are given by:

$$
a_{m}=\frac{n \psi_{m}(n x) \psi_{m}^{\prime}(x)-\psi_{m}(x) \psi_{m}^{\prime}(n x)}{n \psi_{m}(n x) \xi_{m}^{\prime}(x)-\xi_{m}(x) \psi_{m}^{\prime}(n x)}
$$

and

$$
b_{m}=\frac{\psi_{m}(n x) \psi_{m}^{\prime}(x)-n \psi_{m}(x) \psi_{m}^{\prime}(n x)}{\psi_{m}(n x) \xi_{m}^{\prime}(x)-n \xi_{m}(x) \psi_{m}^{\prime}(n x)}
$$

respectively, where $\psi_{m}$ and $\xi_{m}$ are the Riccati-Bessel functions related to the spherical Bessel functions by $\psi_{m}(z)=$ $z j_{m}(z)$ and $\xi_{m}(z)=z h_{m}^{(1)}(z)$ [4], [11]. The prime (') implies differentiation with respect to the arguments and is given in [12] by:

$$
\left(\frac{1}{z} \frac{d}{d z}\right)^{m}\left[z^{n+1} f_{n}(z)\right]=z^{n-m+1} f_{n-m}(z)
$$

where $f_{n}(z)$ is any of the spherical Bessel functions.

Ref. [4] defines $n$ in (4) and (5) as the ratio of the complex refractive index of the particle to the real refractive index of the host medium $n_{0}$ while [5] defines it as just the refractive index of the particle. Also, the size parameter, $x$, is defined in [4] as $k_{0} a n_{0}$, and in [5] as $k_{0} a$, where $a$ is the sphere's radius. The effect of the electric and magnetic fields on the particles is equivalent to dipoles having effective electric, $\alpha_{1}$ and magnetic, $\beta_{1}$ dipole polarisabilities [1], [4], accounting for each of the multipole terms present in the scattered field, as depicted in the scattering coefficients.

Both studies quote the Clausius-Mossotti equation for effective $\varepsilon$ and $\mu$ of the mixture differently; [4] states it as:

$$
\varepsilon_{e f f}=1+\frac{4 \pi N \alpha_{1}}{1-4 \pi N \alpha_{1} / 3}, \mu_{e f f}=1+\frac{4 \pi N \beta_{1}}{1-4 \pi N \beta_{1} / 3}
$$

where $N$ is the number of particles per unit volume related to the volume fraction by $f_{v}=4 \pi N a^{3} / 3, \alpha_{1}=\frac{3 i a_{1}}{2 k_{0}^{3} n_{0}^{3}}$, and $\beta_{1}=$ $\frac{3 i b_{1}}{2 k_{0}^{3} n_{0}^{3}}$; while [5] does not have the ' $4 \pi$ ' multiplier in its equation for effective $\varepsilon$ and $\mu$. Also, [5] represents the polarisabilities as $\alpha_{1}=\frac{6 \pi i a_{1}}{k_{0}^{3}}, \beta_{1}=\frac{6 \pi i b_{1}}{k_{0}^{3}}$. Just the first term of the coefficients' series is retained because it is assumed that the particle radius is much less than the wavelength in the host medium. Another difference in these analyses is that the EM properties of the host was accounted for in [4] but not in [5]. However, they both follow the condition that the spacing and radius should be smaller than the wavelength. The example in [5] was reproduced (not shown).

\section{Other Contributors [6]-[8]}

The analysis in [6] examines "identical conducting obstacles in a regular three-dimensional pattern", held by a lightweight binder, as an artificial dielectric. The formulas derived for the effective $\varepsilon$ and $\mu$ of the mixture use Lorentz Theory, assume symmetrical obstacles and a cubical lattice pattern, consider only dipole interactions and is valid only where the size of the obstacles are smaller than their spacing. These formulas are:

$$
\varepsilon_{r}=1+\frac{N \alpha_{e}}{1-\alpha_{e} C} ; \text { and } \mu_{r}=1+\frac{N \alpha_{m}}{1-\alpha_{m} C}
$$

where $\varepsilon_{r}$ and $\mu_{r}$ are the effective permittivity and permeability of the mixture, $\alpha_{e}, \alpha_{m}$ are the obstacle's electric and magnetic polarisabilities respectively given as a function of its size and shape, $N$ is defined as above and $C$ is the interaction constant given by:

$$
C=\frac{1.202}{\pi b^{3}}-\frac{8 \pi}{b^{3}}\left[K_{0}\left(\frac{2 \pi c}{b}\right)+K_{0}\left(\frac{2 \pi a}{b}\right)\right]
$$

where $a, b$ and $c$ are the spacing of the obstacles in the $x, y$ and $z$ directions respectively, $K_{0}(\cdot)$ is the modified Bessel function of the second kind.

The approximation for effective $\varepsilon$ and $\mu$ for metal particles in [1] is the same as that for spherical obstacles in [6] when $\alpha_{e}, \alpha_{m}, N$ and $C$ are substituted with their equivalent equations.

Reference [7] starts from first principles and derives the expressions for effective $\varepsilon$ and $\mu$ as

$$
\kappa_{\text {eff }}=\kappa_{e}+\frac{n \alpha}{1-\frac{n \alpha}{3 \kappa_{e}}}
$$

where $\alpha$ is the dipole polarisability given by $\alpha=$ $V\left(\kappa_{i}-\kappa_{e}\right) 3 \kappa_{e} /\left(\kappa_{i}+2 \kappa_{e}\right), n$ is number density of the spheres $\left(\mathrm{m}^{-3}\right), \kappa_{e}, \kappa_{i}$ are the host and inclusion's permittivity (or permeability) respectively, and $V$ is the volume of the sphere. Equation (10) quoted as the Clausius-Mossotti equation, differs from (7) by the presence of the host's properties in it and can be reordered to give an equation similar to those in [1]. Common to these two analyses is the absence of any frequency-dependent term in their equations.

Reference [8] extended the work in [7] by adding that the number densities, electric and magnetic polarisabilities of the 
spheres will differ for electric and magnetic resonance modes, and investigates them by using two spheres of different radii.

\section{GRAPHICAL RESULTS}

The formulas from these reviewed authors [1], [4]-[8] were compared with respect to frequency. The values of the EM parameters shown in the graphs are all relative values. Data used: $a=50 \mu \mathrm{m}, s=100 \mu \mathrm{m}$, permittivity of the particle, $\varepsilon_{2}=$ $4.9(1-\mathrm{j} 0.025)$ (FR4), permittivity of host, $\varepsilon_{l}=1.05$ (Polyfoam), permeability of host, $\mu_{1}=\mu_{2}=1$, frequency range: $1-500 \mathrm{GHz}$.

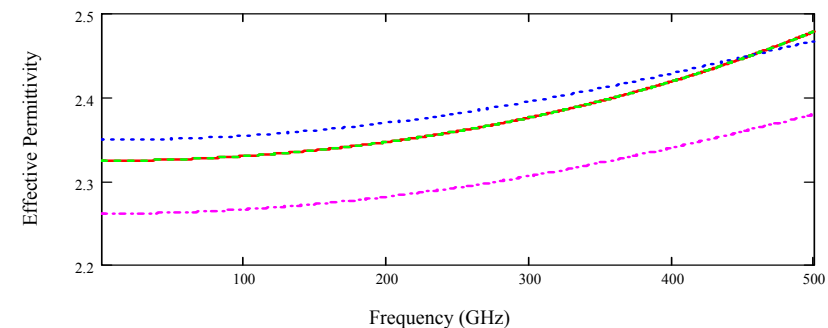

(a)

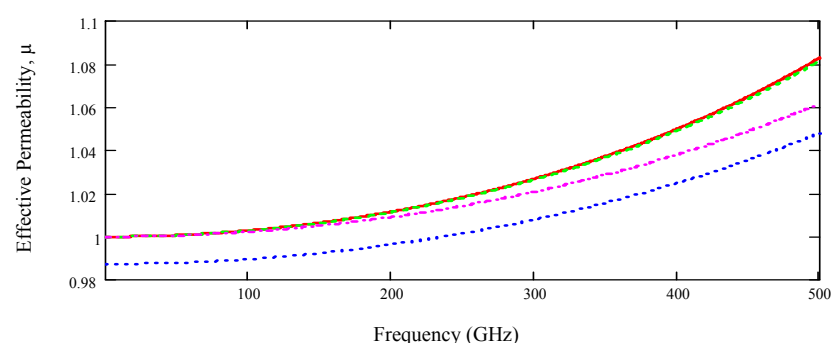

(b)

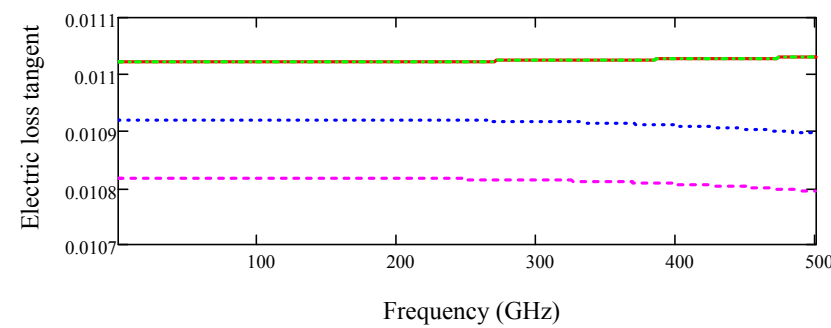

(c)

Fig. 2 Representation of effective (a) permittivity, $\varepsilon$, (b) permeability, $\mu$ and (c) electric loss tangent of a mixture by $[1](-),[4](\cdots),[5](-\cdot-\cdot)$ and [8] (---)

The values shown above in Fig. 2 are the absolute values of the parameters. From these graphs, the representations by [1] and [8] agree while that of [4] and [5] produce different values albeit with the same trend. References [4] and [5] typically agree when $\varepsilon_{1}=1$. If this value is changed, values from [5] will remain the same while those from [4] will change accordingly.

\section{A. Further Studies}

In order to understand how the different components of the defining equations affect the effective parameters of the mixture, parametric studies were carried out.

1) Particle Size, $a$ : Keeping frequency at $1 \mathrm{GHz}$, spacing, $s$ at $1 \mu \mathrm{m}$, while varying the radius of the particle from 0.1 to
$0.5 \mu \mathrm{m}$, the effective $\varepsilon$ and $\mu$ of a mixture with (a) an air and (b) a dielectric host medium was obtained as shown in Fig. 3 below. Even though $\mu_{1}=\mu_{2}=1$, effective $\mu$ reduces with increase in the particle volume fraction. The permittivity of the metal (in this case, Copper) was obtained from first principles using the Drude model [13].

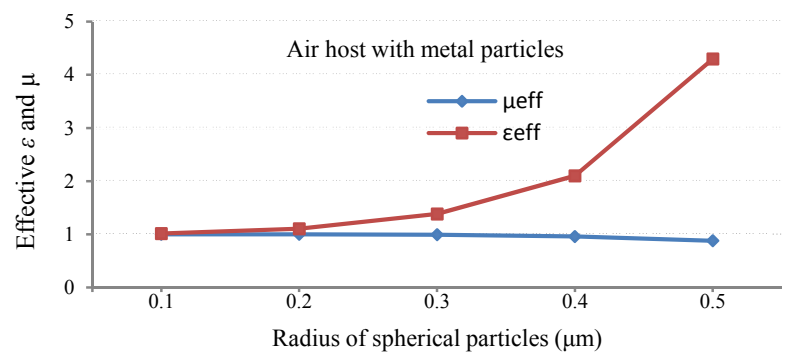

(a)

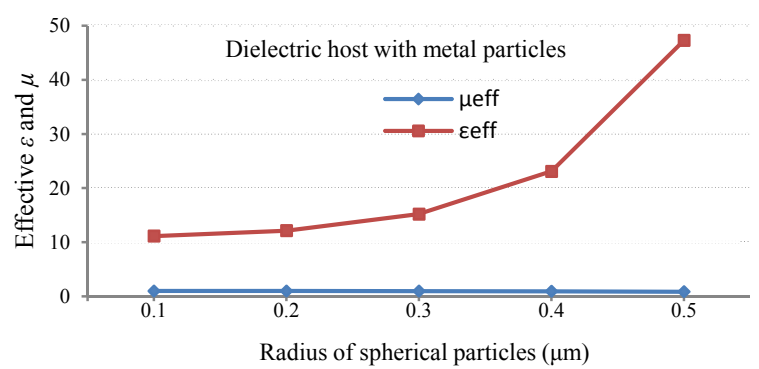

(b)

Fig. 3 Variation of effective $\varepsilon$ and $\mu$ with particle radius for (a) air $\left(\varepsilon_{l}=1\right)$ and (b) dielectric $\left(\varepsilon_{1}=11(1-\mathrm{j} 0.0028)\right) \cdot \mu_{1}=\mu_{2}=1$.

The equations from [1], [4] and [8] were used to obtain the values used in Fig. 3 above. These values did not vary significantly from each other and thus a near-average was used in the graphs. However, [5] varied by nearly a multiple of the $\varepsilon$ and $\mu$ of the host medium, thus only agreeing when the host was air.

2) Particle Spacing, $s$ : With frequency at $1 \mathrm{GHz}$, particle size of $5 \mu \mathrm{m}$, the spacing was varied from $10-30 \mu \mathrm{m}$ using equations from [1], [4]-[8].

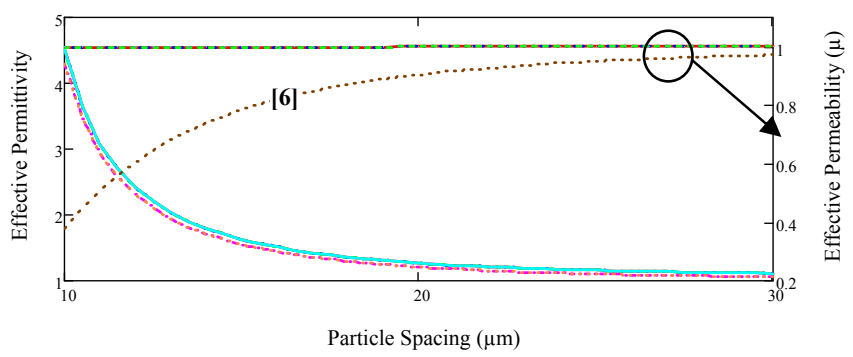

Fig. 4 Variation of effective $\varepsilon$ and $\mu$ with particle spacing for dielectric host, $\varepsilon_{1}=1.05$, and metal particle, $\varepsilon_{2}=(1.27-\mathrm{j} 103.53) \cdot 10^{6}$

Fig. 4 shows that all the formulations agree except for the effective permeability obtained from [6] (‥) in which effective $\mu$ increases to 1 with increase in spacing. 
3) Particle Permittivity, $\varepsilon_{2}$ : The effect of changing the permittivity of the particle is changed from 1 to 200, while keeping $a=5 \mu \mathrm{m}, s=15 \mu \mathrm{m}, \varepsilon_{1}=1.05$ and frequency at $1 \mathrm{GHz}$, is shown below in Fig. 5, using equations in [1], [4], [5], [8].

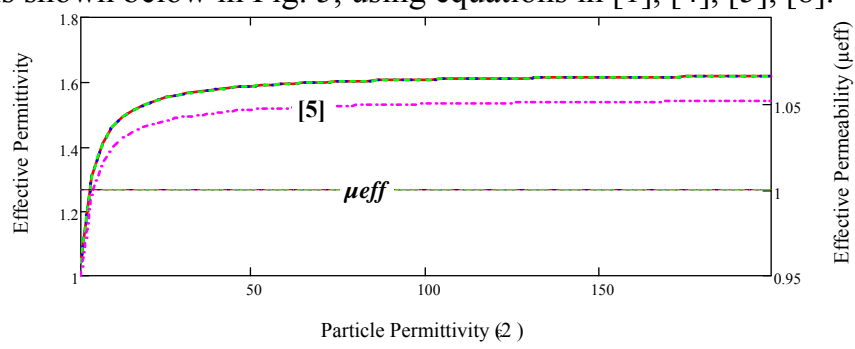

Fig. 5 Variation of effective $\varepsilon$ and $\mu$ with particle permittivity for $\varepsilon_{l}=1.05$

The result from [5] for the effective $\varepsilon$ varies significantly from others as it does not account for the EM parameters of the host in its equations.

From these results, equations from [1] and [8] agree but differ slightly from that of [4] and [5]. Formulations from [4] seem to provide a more thorough analysis by the use of Bessel functions in its analysis, and takes into account all the dimensions and EM properties of the host and the inclusions. The results obtained are more closely in agreement with that obtained from [1] and [8] which are approximations of the Bessel functions for their magnetic and electric scattering coefficients. Thus equations from [1] or [8] and [4] will be used to a greater extent than those from [5], [6] or [7].

\section{CONCLUSIONS}

Effective medium theories for obtaining the permittivity and permeability of mixtures containing evenly spaced spherical particles in a homogenous host medium has been examined for how they compared for different authors. The factors affecting these parameters have been studied for their individual effects. Some of the results from the reviewed papers were reproducible (although not included in this paper).

Future work will involve running FDTD (Finite Difference Time Domain) simulations of heterogeneous mixtures and using Inverse Scattering formulations [14] to extract their effective $\varepsilon$ and $\mu$. Subsequently, these structures will be built, tested and measured to compare with the mathematical analysis and simulation results.

\section{REFERENCES}

[1] L. Lewin, "The electrical constants of a material loaded with spherical particles", IEE - Part III: Radio and Communication Engineering, vol. 94, issue 27, pp. 65-68, Jan. 1947

[2] L. Rayleigh, "On the influence of obstacles arranged in rectangular order on the properties of a medium," Phil. Mag., ser. 5, vol. 34, pp.481-502, 1892

[3] W. T. Doyle, "The Clausius-Mossotti problem for cubic array of spheres", Journal of Applied Physics, vol. 49, issue 2, pp. 795-797, Feb. 1978

[4] W. T. Doyle, "Optical properties of a suspension of metal spheres", Physics Review B, vol. 39, issue 14, pp. 9852-9858, May 1989.

[5] X. Cai, R. Zhu, and G. Hu, "Experimental study for Metamaterials based on dielectric resonators and wire frame", Metamaterials, vol. 2, issue 4, pp. 220-226, Sept. 2008

[6] R. E. Collin, Ch. 12 in Field Theory of Guided Waves, $2^{\text {nd }}$ edition, IEEE Press: NY, 1991

[7] A. Sihvola, Ch. 3 in Electromagnetic mixing formulas and applications, IEE Electromagnetic Waves Series 47, 1999

[8] I. A. Kolmakov, L. Jylha, S. A. Tretyakov, and S. Maslovski, "Lattice of dielectric particles with double negative response", 28th General Assembly of International Union of Radio Science (URSI), New Delhi, India, paper BCD.5 (0109).pdf, 2005

[9] C. L. Holloway, E. F. Kuester, J. Baker-Jarvis, and P. Kabos, “ A double negative (DNG) composite medium composed of Magnetodielectric spherical particles embedded in a matrix", IEEE Transactions on Antennas and Propagation, vol. 51, no. 10, pp. 25962603, Oct. 2003

[10] W. G. Whittow, and J. C. Vardaxoglou, "Modifying conventional microwave antenna designs using fine scale structures and nanomaterials," LAPC 2009, pp. 749-752, Dec. 2009

[11] J. A. Stratton, pp. $563-565$, Ch. 9 in Electromagnetic Theory, McGraw-Hill Book Inc., 1941

[12] M. Abramowitz, and I. A. Stegun, Ch. 10 in Handbook of Mathematical Functions, $1^{\text {st }}$ edition, Dover Publications, Inc: NY, 1970

[13] M. A. Ordal, R. J. Bell, R. W. Alexander, L. L. Long, and M. R. Query, "Optical properties of fourteen metals in the infrared and far infrared: $\mathrm{Al}, \mathrm{Co}, \mathrm{Cu}, \mathrm{Au}, \mathrm{Fe}, \mathrm{Pb}, \mathrm{Mo}, \mathrm{Ni}, \mathrm{Pd}, \mathrm{Pt}, \mathrm{Ag}, \mathrm{Ti}, \mathrm{V}$, and W," Applied Optics, vol. 24, no. 24, Dec. 1985

[14] N. G. Alexopoulos, C. A. Kyriazidou, and H. F. Contopanagos, "Effective Parameters for Metamorphic Materials and Metamaterials Through a Resonant Inverse Scattering approach," IEEE Transactions on Microwave Theory and Techniques, vol. 55, no. 2, Feb. 2007 